\title{
ANALYTICAL AND NUMERICAL SOLUTIONS FOR TIME-FRACTIONAL NEW COUPLED MKDV EQUATION ARISING IN INTERACTION OF TWO LONG WAVES
}

\author{
ORKUN TASBOZAN ${ }^{1}$, MEHMET ŞENOL $^{2}$, ALI KURT ${ }^{3, *}$, DUMITRU BALEANU $^{4,5}$ \\ ${ }^{1}$ Department of Mathematics, Faculty of Science and Art, Mustafa Kemal University, Hatay, Turkey \\ ${ }^{2}$ Department of Mathematics, Faculty of Science and Art, Nevşehir University, Nevşehir, Turkey \\ ${ }^{3}$ Department of Mathematics, Faculty of Science and Art, Pamukkale University, Denizli, Turkey \\ ${ }^{4}$ Department of Mathematics and Computer Sciences, Faculty of Art and Sciences, Cankaya University, Ankara, Turkey \\ ${ }^{5}$ Institute of Space Sciences, Magurele-Bucharest, Romania \\ *Corresponding Author: pau.dr.alikurt@gmail.com \\ Received Apr. 19, 2019
}

\begin{abstract}
Aвstract. The aim of this paper is to present new exact solution sets of nonlinear conformable time-fractional new coupled $\mathrm{mKdV}$ equations which arise in interaction of two long waves with different dispersion relations by means of sub-equation method. In addition, we also propose an analytical-approximate method namely residual power series method (RPSM) for the system. The fractional derivatives have been explained in newly defined conformable type, during the solution procedure. The exact solutions of the system obtained by the sub-equation method have been compared to approximate solutions derived by RPSM. The results showed that both methods are robust, dependable, easy to apply and a good alternative for seeking solutions of fractional partial differential equations.
\end{abstract}

2010 Mathematics Subject Classification. 35R11; 35A20; 35C05.

Key words and phrases. Conformable fractional derivative, Sub-equation method, Residual power series method, New Coupled mKdV Equation.

\section{INTRODUCTION}

The fractional differential equations have been widely used by more researchers to model real-world problems in recent years. It has been broadly studied and applied for various models in many branches of engineering and science such as dynamical systems [10], mathematical physics [34], fluid mechanics [28], biology [19], viscoelasticity [9] and control [35]. In addition, the investigations for analytical and approximate solutions of fractional partial differential equations (FPDEs) gives scientists the opportunity to define phenomena in applied sciences. Therefore, obtaining analytical and approximate solutions of FPDEs has significant and a special place in above cited fields. Some o the classical analytical and approximate methods for solving fractional differential equations (FDEs) are finite difference method (FDM) [27,32], Adomian decomposition method (ADM) [17,20],

DOI: $10.28924 /$ APJM/6-13 
variational iteration method (VIM) [29], homotopy analysis method (HAM) [24,25] and perturbation-iteration algorithm $[30,31]$.

In this article, the sub-equation [11,36] and residual power series methods [4-7] have been implemented to receive new exact and approximate solutions of time-fractional new coupled $\mathrm{mKdV}$ equations of the form [12]:

$$
\begin{array}{r}
\partial_{t}^{\alpha} u-\frac{1}{2} u_{x x x}+3 u^{2} u_{x}-3\left(v v_{x}\right)_{x}-3\left(u v^{2}\right)_{x}=0, \\
\partial_{t}^{\alpha} v+v_{x x x}-6 u v u_{x}+3\left(v u_{x}\right)_{x}-\left(u^{2}-v^{2}\right) v_{x}=0
\end{array}
$$

The sub-equation method is a powerful tool for obtaining exact solutions of nonlinear FPDEs. It transforms the given system to an ordinary differential equation to solve it easily. Residual power series is also set up on the power series expansion. It may be applied to the equation directly in the absence of discretization, linearization or any transformation by choosing proper initial conditions.

The remainder of the study is organized as follows. Brief explanations of the methods has firstly been given and the implementation of the considered methods are presented on an example to show efficiency and reliability of the proposed methods. Also figures and tables have been presented in order to compare their numerical results. Finally, we discussed about the obtained results as a conclusion.

\section{Preliminaries}

There are a few definition of fractional derivative of order $\alpha>0$. The most widely used are the RiemannLiouville and Caputo fractional derivatives.

Definition 2.1. The Riemann-Liouville fractional derivative operator $D^{\alpha} f(x)$ defined as $[2,15,16]$ :

$$
D^{\alpha} f(x)=\frac{d^{q}}{d x^{q}}\left[\frac{1}{\Gamma(q-\alpha)} \int_{\alpha}^{x} \frac{f(t)}{(x-t)^{\alpha+1-q}} d t\right]
$$

where $\alpha>0$ and $q-1<\alpha<q$.

Definition 2.2. The Caputo fractional derivative of order $\alpha$ defined as [13]:

$$
D_{*}^{\alpha} f(x)=J^{n-\alpha} D^{n} f(x)=\frac{1}{\Gamma(n-\alpha)} \int_{\alpha}^{x}(x-t)^{n-\alpha-1}\left(\frac{d}{d t}\right)^{n} f(t) d t
$$

where $\alpha>0$ for $n \in \mathbb{N}, n-1<\alpha<n$.

Recently, a new definition has been proposed by Khalil et al. [22] which is called "conformable fractional derivative".

Definition 2.3. An $\alpha$-th order "conformable fractional derivative" of a function $f:[0, \infty) \rightarrow \mathbb{R}$ defined by

$$
T_{\alpha}(f)(t)=\lim _{\varepsilon \rightarrow 0} \frac{f\left(t+\varepsilon t^{1-\alpha}\right)-f(t)}{\varepsilon}
$$

for all $\alpha \in(0,1)$ and $t>0$.

The following theorem gives the properties of this new definition [22]. 
Theorem 2.1. Let $f$ and $g$ are $\alpha$-differantiable functions for $\alpha \in(0,1]$ and $t>0$. In that case

1. $T_{\alpha}(m f+n g)=m T_{\alpha}(f)+n T_{\alpha}(g)$ for all $m, n \in \mathbb{R}$,

2. $T_{\alpha}\left(t^{p}\right)=p t^{p-\alpha}$ for all $p$,

3. $T_{\alpha}(f . g)=f T_{\alpha}(g)+g T_{\alpha}(f)$,

4. $T_{\alpha}\left(\frac{f}{g}\right)=\frac{g T_{\alpha}(f)-f T_{\alpha}(g)}{g^{2}}$,

5. $T_{\alpha}(c)=0$ for all $f(t)=c$ constant functions,

6. $T_{\alpha}(f)(t)=t^{1-\alpha} \frac{d f(t)}{d t}$, if $f$ is differentiable.

Definition 2.4. The conformable partial derivatives of an $f$ function of order $\alpha \in(0,1]$ with $x_{1}, \ldots, x_{n}$ variables, is defined as $[8]$

$$
\frac{d^{\alpha}}{d x_{i}^{\alpha}} f\left(x_{1}, \ldots, x_{n}\right)=\lim _{\varepsilon \rightarrow 0} \frac{f\left(x_{1}, \ldots, x_{i-1}, x_{i}+\varepsilon x_{i}^{1-\alpha}, \ldots, x_{n}\right)-f\left(x_{1}, \ldots, x_{n}\right)}{\varepsilon} .
$$

Definition 2.5. The conformable integral of an $f$ function for $a \geqslant 0$ is defined as [33]

$$
I_{\alpha}^{a}(f)(s)=\int_{a}^{s} \frac{f(t)}{t^{1-\alpha}} d t .
$$

In this section, some important definitions and theorems about residual power series will be given.

Theorem 2.2. Suppose that $f$ is an infinitely $\alpha$-differentiable function at a neigborhood of a point $t_{0}$ for some $0<\alpha \leq 1$, then $f$ has the fractional power series expansion of the form:

$$
f(t)=\sum_{k=0}^{\infty} \frac{\left(T_{\alpha}^{t_{0}} f\right)^{(k)}\left(t_{0}\right)\left(t-t_{0}\right)^{k \alpha}}{\alpha^{k} k !}, t_{0}<t<t_{0}+R^{\frac{1}{\alpha}}, R>0 .
$$

Here $\left(T_{\alpha}^{t_{0}} f\right)^{(k)}\left(t_{0}\right)$ represents the application of the fractional derivative $k$-times [1].

Definition 2.6. A multiple fractional power series about $t_{0}=0$ is defined by $\sum_{n=0}^{\infty} f_{n}(x) t^{n \alpha}$ for $0 \leq m-1<\alpha<m$, where $t$ is a variable and $f_{n}(x)$ are functions called the coefficients of the series $[3,18]$.

Theorem 2.3. Assume that $u(x, t)$ has a multiple fractional power series representation at $t_{0}=0$ of the form [3]

$$
u(x, t)=\sum_{n=0}^{\infty} f_{n}(x) t^{n \alpha}, 0 \leq m-1<\alpha<m, x \in I, 0 \leq t \leq R^{\frac{1}{\alpha}} .
$$

If $u_{t}^{(n \alpha)}(x, t), n=0,1,2, \ldots$ are continuous on $I \times\left(0, R^{\frac{1}{\alpha}}\right)$, then $f_{n}(x)=\frac{u_{t}^{(n \alpha)}(x, 0)}{\alpha^{n} n !}$.

\section{Basics of the Sub-Equation Method}

In this section, we present a short explanation of sub equation method [36]. For a given conformable fractional differential equation with two independent variables $x$ and $t$, consider

$$
P\left(u, D_{t}^{\alpha} u, D_{x} u, D_{t}^{2 \alpha} u, D_{x}^{2} u, \ldots\right)=0
$$


where $D_{t}^{\alpha} u$ is conformable fractional derivatives of unknown function $u(x, t)$ and $D_{t}^{2 \alpha}$ stand for two times conformable fractional derivative of it. By adopting the wave transformation [14]

$$
u(x, t)=U(\xi), \xi=x-k \frac{t^{\alpha}}{\alpha}
$$

and chain rule [1], Eq. (3.1) turns into nonlinear ordinary differential equation

$$
G\left(U ; U^{\prime} ; U^{\prime \prime} ; \ldots\right)=0
$$

where primes show integer order derivatives with respect to new wave variables $\xi$ and $k$ that will be examined later. We assume that equation (3.3) has a solution in the form

$$
U(\xi)=\sum_{i=0}^{N} a_{i} \varphi^{i}(\xi), a_{N} \neq 0
$$

where $a_{i}(0 \leq i \leq N)$ are constant coefficients to be determined later. $N$ is a positive integer which is going to be calculated by balancing the highest order derivatives of linear and nonlinear terms [26] in equation (3.3) and $\varphi(\xi)$ is a solution of Riccati equation

$$
\varphi^{\prime}(\xi)=\sigma+(\varphi(\xi))^{2}
$$

where $\sigma$ is a constant. Some special solutions for the Riccati equation (3.5) are given by the following formulas.

$$
\varphi(\xi)=\left\{\begin{array}{cc}
-\sqrt{-\sigma} \tanh (\sqrt{-\sigma} \xi), & \sigma<0 \\
-\sqrt{-\sigma} \operatorname{coth}(\sqrt{-\sigma} \xi), & \sigma<0 \\
\sqrt{\sigma} \tan (\sqrt{\sigma} \xi), & \sigma>0 \\
\sqrt{\sigma} \cot (\sqrt{\sigma} \xi), & \sigma>0 \\
-\frac{1}{\xi+\varpi}, \varpi i s \text { a cons., } & \sigma=0
\end{array}\right.
$$

Subrogating equations (3.4) and (3.5) into equation (3.3) we obtain a polynomial with respect $\varphi(\xi)$. Setting all the coefficients of $\varphi^{i}(\xi)$ to zero, one gets nonlinear algebraic system in $k, a_{i}(i=0,1, \ldots, N)$. By solving these nonlinear algebraic system we determine the constants $k, a_{i}(i=0,1, \ldots, N)$. Then substituting obtained constants from the nonlinear algebraic system and the solutions of equation (3.5) into equation (3.4) by the help of the formulas (3.6) we acquire the exact solutions for equation (3.1).

\section{BASICS OF THE RESIDUAL POWER SERIES METHOD}

To illustrate the basic idea of RPSM, let's take the following nonlinear fractional differential equation [23]:

$$
T_{\alpha} u(x, t)+N[x] u(x, t)+L[x] u(x, t)=c(x, t),
$$

where $n-1<n \alpha \leq n, x \in \mathbb{R}, t>0$ and given with the initial condition

$$
f_{0}(x)=u(x, 0)=f(x)
$$

Here, $L[x]$ is a linear, $N[x]$ is a non-linear operator and $c(x, t)$ are continuous functions. 
The RPSM method made up of stating the solution of the equation (4.1) subject to (4.2) as a fractional power series expansion around $t=0$.

$$
f_{(n-1)}(x)=T_{t}^{(n-1) \alpha} u(x, 0)=h(x)
$$

The expansion form of the solution is denoted by

$$
u(x, t)=f(x)+\sum_{n=1}^{\infty} f_{n}(x) \frac{t^{n \alpha}}{\alpha^{n} n !} .
$$

In the next step, the $k$.truncted series of $u(x, t)$, namely $u_{k}(x, t)$ can be written as:

$$
u_{k}(x, t)=f(x)+\sum_{n=1}^{k} f_{n}(x) \frac{t^{n \alpha}}{\alpha^{n} n !} .
$$

Since the 1 st approximate solution $u_{1}(x, t)$ is

$$
u_{1}(x, t)=f(x)+f_{1}(x) \frac{t^{\alpha}}{\alpha^{n}}
$$

then $u_{k}(x, t)$ might be reformulated as

$$
u_{k}(x, t)=f(x)+f_{1}(x) \frac{t^{\alpha}}{\alpha^{n}}+\sum_{n=2}^{k} f_{n}(x) \frac{t^{n \alpha}}{\alpha^{n} n !}, k=2,3,4, \ldots
$$

for $0<\alpha \leq 1,0 \leq t<\mathbb{R}^{\frac{1}{v}}, x \in I$.

Initially we express the residual function and the $k-t h$ residual function

$$
\begin{gathered}
\operatorname{Resu}(x, t)=T_{\alpha} u(x, t)+N[x] u(x, t)+L[x] u(x, t)-c(x, t), \\
\operatorname{Resu}_{k}(x, t)=T_{\alpha} u_{k}(x, t)+N[x] u_{k}(x, t)+L[x] u_{k}(x, t)-g(x, t), k=1,2,3, \ldots
\end{gathered}
$$

respectively. Obviously, $\operatorname{Res}(x, t)=0$ and $\lim _{k \rightarrow \infty} \operatorname{Resu} u_{k}(x, t)=\operatorname{Resu}(x, t)$ for each $x \in I$ and $t \geq 0$. Indeed this bring about $\frac{\partial^{(n-1) \alpha}}{\partial t^{(n-1) \alpha}} \operatorname{Resu}_{k}(x, t)=0$ for $n=1,2,3, \ldots, k$. Since the fractional derivative of a constant is zero in the conformable sense $[4,21]$. Solving the equation $\frac{\partial^{(n-1) \alpha}}{\partial t^{(n-1) \alpha}} \operatorname{Res} u_{k}(x, 0)=0$ gives us the required $f_{n}(x)$ coefficients. So the $u_{n}(x, t)$ approximate solutions can be obtained respectively in this fashion.

\section{Implementation of the Considered Methods}

5.1. Wave Solutions for Fractional New Coupled mKdV Equation. Let consider the fractional new coupled $\mathrm{mKdv}$ equation in Eq. (1.1) where $t \geq 0,0<\alpha \leq 1$ and the derivatives are in conformable sense. With the aid of the chain rule [1] with the aid of wave transform $\xi=x-k \frac{t^{\alpha}}{\alpha}[14]$ led to

$$
\begin{gathered}
-k U-\frac{1}{2} U^{\prime \prime}+U^{3}-3 V V^{\prime}-3 U V^{2}=0, \\
-k V+V^{\prime \prime}+V^{3}-3 V U^{\prime}-3 V U^{2}=0 .
\end{gathered}
$$


Using balancing procedure in equation (5.1), we obtain $N=1$. So we set up

$$
\begin{gathered}
U(\xi)=a_{0}+a_{1} \varphi(\xi), \\
V(\xi)=b_{0}+b_{1} \varphi(\xi)
\end{gathered}
$$

where $\varphi(\xi)$ satisfies the Riccati equation (3.5). Subrogating equations (5.2) with equation (3.5) into eq. (5.1), collecting and equating all the coefficients of $\varphi^{i}(\xi)$ to zero yield a set of algebraic equations with respect to $a_{0}, a_{1}, b_{0}, b_{1}, k$. These equations can be mentioned

$$
\begin{aligned}
& \varphi^{0}: a_{0}^{3}-3 a_{0} b_{0}^{2}-a_{0} k-3 b_{0} b_{1} \sigma=0,-3 a_{0}^{2} b_{0}+b_{0}^{3}-b_{0} k+3 a_{1} b_{0} \sigma=0, \\
& \varphi^{1}: 3 a_{0}^{2} a_{1}-3 a_{1} b_{0}^{2}-6 a_{0} b_{0} b_{1}-a_{1} k-a_{1} \sigma-3 b_{1}^{2} \sigma=0,-6 a_{0} a_{1} b_{0}-3 a_{0}^{2} b_{1}+3 b_{0}^{2} b_{1}-b_{1} k+2 b_{1} \sigma+3 a_{1} b_{1}=0, \\
& \varphi^{2}: 3 a_{0} a_{1}^{2}-3 b_{0} b_{1}-6 a_{1} b_{0} b_{1}-3 a_{0} b_{1}^{2}=0,3 a_{1} b_{0}-3 a_{1}^{2} b_{0}-6 a_{0} a_{1} b_{1}+3 b_{0} b_{1}^{2}=0, \\
& \varphi^{3}:-a_{1}+a_{1}^{3}-3 b_{1}^{2}-3 a_{1} b_{1}^{2}=0,2 b_{1}+3 a_{1} b_{1}-3 a_{1}^{2} b_{1}+b_{1}^{3}=0 .
\end{aligned}
$$

Solving the above system equations with the help of computer software Mathematica, we get

$$
a_{0}=0, a_{1}=-\frac{1}{2}, b_{0}=0, b_{1}=\frac{1}{2}, k=\frac{\sigma}{2}
$$

Using the values of above coefficients and the equalities (3.6) along with the wave transform $\xi=x-k \frac{t^{\alpha}}{\alpha}$ and (5.2) yields the following travelling wave solutions

$$
\begin{aligned}
& u_{1}(x, t)=\frac{1}{2} \sqrt{-\sigma} \tanh \left(\sqrt{-\sigma}\left(x-\frac{\sigma t^{\alpha}}{2 \alpha}\right)\right), \\
& v_{1}(x, t)=-\frac{1}{2} \sqrt{-\sigma} \tanh \left(\sqrt{-\sigma}\left(x-\frac{\sigma t^{\alpha}}{2 \alpha}\right)\right), \\
& u_{2}(x, t)=\frac{1}{2} \sqrt{-\sigma} \operatorname{coth}\left(\sqrt{-\sigma}\left(x-\frac{\sigma t^{\alpha}}{2 \alpha}\right)\right), \\
& v_{2}(x, t)=-\frac{1}{2} \sqrt{-\sigma} \operatorname{coth}\left(\sqrt{-\sigma}\left(x-\frac{\sigma t^{\alpha}}{2 \alpha}\right)\right), \\
& u_{3}(x, t)=-\frac{1}{2} \sqrt{\sigma} \tan \left(\sqrt{\sigma}\left(x-\frac{\sigma t^{\alpha}}{2 \alpha}\right)\right), \\
& v_{3}(x, t)=\frac{1}{2} \sqrt{\sigma} \tan \left(\sqrt{\sigma}\left(x-\frac{\sigma t^{\alpha}}{2 \alpha}\right)\right), \\
& u_{4}(x, t)=\frac{1}{2} \sqrt{\sigma} \cot \left(\sqrt{\sigma}\left(x-\frac{\sigma t^{\alpha}}{2 \alpha}\right)\right), \\
& v_{4}(x, t)=-\frac{1}{2} \sqrt{\sigma} \cot \left(\sqrt{\sigma}\left(x-\frac{\sigma t^{\alpha}}{2 \alpha}\right)\right) .
\end{aligned}
$$

5.2. Approximate Solutions for Fractional New Coupled $\mathbf{m K d V}$ Equation. Consider the nonlinear time-fractional

new coupled $\mathrm{mKdV}$ equation in Eq. (1.1) with the following initial equations

$$
\begin{aligned}
& u(x, 0)=f(x)=\frac{1}{2} \sqrt{-\sigma} \tanh (\sqrt{-\sigma} x), \\
& v(x, 0)=g(x)=-\frac{1}{2} \sqrt{-\sigma} \tanh (\sqrt{-\sigma} x) .
\end{aligned}
$$


For residual power series

$$
\begin{aligned}
& u(x, t)=f(x)+\sum_{n=1}^{\infty} f_{n}(x) \frac{t^{n \alpha}}{\alpha^{n} n !}, \\
& v(x, t)=g(x)+\sum_{n=1}^{\infty} g_{n}(x) \frac{t^{n \alpha}}{\alpha^{n} n !}
\end{aligned}
$$

and $k$.truncated series of $u(x, t)$ and $v(x, t)$

$$
\begin{aligned}
& u_{k}(x, t)=f(x)+\sum_{n=1}^{k} f_{n}(x) \frac{t^{n \alpha}}{\alpha^{n} n !}, k=1,2,3, \ldots, \\
& v_{k}(x, t)=g(x)+\sum_{n=1}^{k} g_{n}(x) \frac{t^{n \alpha}}{\alpha^{n} n !}, k=1,2,3, \ldots
\end{aligned}
$$

Therefore, the $k$-th residual functions of time-fractional new coupled $\mathrm{mKdV}$ equation are:

$$
\begin{aligned}
& \operatorname{Resu}_{k}(x, t)=\partial_{t}^{\alpha} u_{k}-\frac{1}{2} u_{k, x x x}+3 u_{k}^{2} u_{k, x}-3\left(v_{k} v_{k, x}\right)_{x}-3\left(u_{k} v_{k}^{2}\right)_{x}, \\
& \operatorname{Resv}_{k}(x, t)=\partial_{t}^{\alpha} v_{k}+v_{k, x x x}-6 u_{k} v_{k} u_{k, x}+3\left(v_{k} u_{k, x}\right)_{x}-\left(u_{k}^{2}-v_{k}^{2}\right) v_{k, x} .
\end{aligned}
$$

To determine the coefficients $f_{1}(x)$ and $g_{1}(x)$, in $u_{1}(x, t)$ and $v_{1}(x, t)$, we should replace the $1 s t$ truncated series $u_{1}(x, t)=f(x)+f_{1}(x) \frac{t^{\alpha}}{\alpha}$ and $v_{1}(x, t)=g(x)+g_{1}(x) \frac{t^{\alpha}}{\alpha}$ into the $1 s t$ truncated residual functions as

$$
\begin{aligned}
\operatorname{Resu}_{1}(x, t)= & f_{1}(x)+3\left(f(x)+\frac{t^{\alpha} f_{1}(x)}{\alpha}\right)^{2}\left(f^{\prime}(x)+\frac{t^{\alpha} f_{1}^{\prime}(x)}{\alpha}\right)-3\left(g(x)+\frac{t^{\alpha} g_{1}(x)}{\alpha}\right)^{2} \\
& \times\left(f^{\prime}(x)+\frac{t^{\alpha} f_{1}^{\prime}(x)}{\alpha}\right)+6\left(f(x)+\frac{t^{\alpha} f_{1}(x)}{\alpha}\right)\left(g(x)+\frac{t^{\alpha} g_{1}(x)}{\alpha}\right)\left(g^{\prime}(x)+\frac{t^{\alpha} g_{1}^{\prime}(x)}{\alpha}\right) \\
& -3\left(\left(g^{\prime}(x)+\frac{t^{\alpha} g_{1}^{\prime}(x)}{\alpha}\right)^{2}+\left(g(x)+\frac{t^{\alpha} g_{1}(x)}{\alpha}\right)\left(g^{\prime \prime}(x)+\frac{t^{\alpha} g_{1}^{\prime \prime}(x)}{\alpha}\right)\right) \\
& +\frac{1}{2}\left(-f^{(3)}(x)-\frac{t^{\alpha} f_{1}^{(3)}(x)}{\alpha}\right), \\
\operatorname{Resv}_{1}(x, t)= & g_{1}(x)-6\left(f(x)+\frac{t^{\alpha} f_{1}(x)}{\alpha}\right)\left(g(x)+\frac{t^{\alpha} g_{1}(x)}{\alpha}\right)\left(f^{\prime}(x)+\frac{t^{\alpha} f_{1}^{\prime}(x)}{\alpha}\right) \\
& -3\left(\left(f(x)+\frac{t^{\alpha} f_{1}(x)}{\alpha}\right)^{2}-\left(g(x)+\frac{t^{\alpha} g_{1}(x)}{\alpha}\right)^{2}\right)\left(g^{\prime}(x)+\frac{t^{\alpha} g_{1}^{\prime}(x)}{\alpha}\right) \\
& +3\left(f^{\prime}(x)+\frac{t^{\alpha} f_{1}^{\prime}(x)}{\alpha}\right)\left(g^{\prime}(x)+\frac{t^{\alpha} g_{1}^{\prime}(x)}{\alpha}\right)+\left(g(x)+\frac{t^{\alpha} g_{1}(x)}{\alpha}\right)\left(f^{\prime \prime}(x)+\frac{t^{\alpha} f_{1}^{\prime \prime}(x)}{\alpha}\right) \\
& +g^{(3)}(x)+\frac{t^{\alpha} g_{1}^{(3)}(x)}{\alpha} .
\end{aligned}
$$

Now for the substitution of $t=0$ through equation $\operatorname{Resu}_{1}(x, t)$ and $\operatorname{Resu}_{1}(x, t)$ to obtain

$$
\operatorname{Resu}_{1}(x, 0)=f_{1}(x)+3 f^{2}(x) f^{\prime}(x)-3\left(g^{2}(x) f^{\prime}(x)+2 f(x) g(x) g^{\prime}(x)\right)-3\left(g^{\prime 2}(x)+g(x) g^{\prime \prime}(x)\right)-\frac{1}{2} f^{(3)}(x),
$$

$$
\operatorname{Resv}_{1}(x, 0)=g_{1}(x)-6 f(x) g(x) f^{\prime}(x)-3\left(f^{2}(x)-g^{2}(x)\right) g^{\prime}(x)+3\left(f^{\prime}(x) g^{\prime}(x)+g(x) f^{\prime \prime}(x)\right)+g^{(3)}(x) \text {. }
$$


Thus for $\operatorname{Resu}_{1}(x, 0)=0$ and $\operatorname{Resv}_{1}(x, 0)=0$,

$$
\begin{gathered}
f_{1}(x)=-3 f^{2} f^{\prime}+3 g^{2} f^{\prime}+6 f g g^{\prime}+3 g^{\prime 2}+3 g g^{\prime \prime}+\frac{1}{2} f^{(3)}, \\
g_{1}(x)=6 f g f^{\prime}+3 f^{2} g^{\prime}-3 g^{2} g^{\prime}-3 f^{\prime} g^{\prime}-3 g f^{\prime \prime}-g^{(3)} .
\end{gathered}
$$

Therefore, we obtain the 1st RPS approximate solutions of time-fractional equation as

$$
\begin{aligned}
& u_{1}(x, t)=f+\frac{t^{\alpha}\left(-3 f^{2} f^{\prime}+3 g^{2} f^{\prime}+6 f g g^{\prime}+3 g^{\prime 2}+3 g g^{\prime \prime}+\frac{1}{2} f^{(3)}\right)}{\alpha}, \\
& v_{1}(x, t)=g+\frac{t^{\alpha}\left(6 f g f^{\prime}+3 f^{2} g^{\prime}-3 g^{2} g^{\prime}-3 f^{\prime} g^{\prime}-3 g f^{\prime \prime}-g^{(3)}\right)}{\alpha} .
\end{aligned}
$$

Again, to determine the second unknown coefficients $f_{2}(x)$ and $g_{2}(x)$, we replace the $2 n d$ truncated series solutions $u_{2}(x, t)=f(x)+f_{1}(x) \frac{t^{\alpha}}{\alpha}+f_{2}(x) \frac{t^{2 \alpha}}{2 \alpha^{2}}$ and $v_{2}(x, t)=g(x)+g_{1}(x) \frac{t^{\alpha}}{\alpha}+g_{2}(x) \frac{t^{2 \alpha}}{2 \alpha^{2}}$ into the $2 n d$ truncated residual functions and obtain

$$
\begin{aligned}
& \operatorname{Resu}_{2}(x, t)=f_{1}(x)+\frac{t^{\alpha} f_{2}(x)}{\alpha}+3\left(f(x)+\frac{t^{\alpha} f_{1}(x)}{\alpha}+\frac{t^{2 \alpha} f_{2}(x)}{2 \alpha^{2}}\right)^{2}\left(f^{\prime}(x)+\frac{t^{\alpha} f_{1}^{\prime}(x)}{\alpha}+\frac{t^{2 \alpha} f_{2}^{\prime}(x)}{2 \alpha^{2}}\right) \\
& -3\left(g(x)+\frac{t^{\alpha} g_{1}(x)}{\alpha}+\frac{t^{2 \alpha} g_{2}(x)}{2 \alpha^{2}}\right)^{2}\left(f^{\prime}(x)+\frac{t^{\alpha} f_{1}^{\prime}(x)}{\alpha}+\frac{t^{2 \alpha} f_{2}^{\prime}(x)}{2 \alpha^{2}}\right) \\
& +6\left(f(x)+\frac{t^{\alpha} f_{1}(x)}{\alpha}+\frac{t^{2 \alpha} f_{2}(x)}{2 \alpha^{2}}\right)\left(g(x)+\frac{t^{\alpha} g_{1}(x)}{\alpha}+\frac{t^{2 \alpha} g_{2}(x)}{2 \alpha^{2}}\right) \\
& \times\left(g^{\prime}(x)+\frac{t^{\alpha} g_{1}^{\prime}(x)}{\alpha}+\frac{t^{2 \alpha} g_{2}^{\prime}(x)}{2 \alpha^{2}}\right)-3\left(g^{\prime}(x)+\frac{t^{\alpha} g_{1}^{\prime}(x)}{\alpha}+\frac{t^{2 \alpha} g_{2}^{\prime}(x)}{2 \alpha^{2}}\right)^{2} \\
& +\left(g(x)+\frac{t^{\alpha} g_{1}(x)}{\alpha}+\frac{t^{2 \alpha} g_{2}(x)}{2 \alpha^{2}}\right) \times\left(g^{\prime \prime}(x)+\frac{t^{\alpha} g_{1}^{\prime \prime}(x)}{\alpha}+\frac{t^{2 \alpha} g_{2}^{\prime \prime}(x)}{2 \alpha^{2}}\right) \\
& +\frac{1}{2}\left(-f^{(3)}(x)-\frac{t^{\alpha} f_{1}^{(3)}(x)}{\alpha}-\frac{t^{2 \alpha} f_{2}^{(3)}(x)}{2 \alpha^{2}}\right) \\
& \operatorname{Resv}_{2}(x, t)=g_{1}(x)+\frac{t^{\alpha} g_{2}(x)}{\alpha}-6\left(f(x)+\frac{t^{\alpha} f_{1}(x)}{\alpha}+\frac{t^{2 \alpha} f_{2}(x)}{2 \alpha^{2}}\right)\left(g(x)+\frac{t^{\alpha} g_{1}(x)}{\alpha}+\frac{t^{2 \alpha} g_{2}(x)}{2 \alpha^{2}}\right) \\
& \times\left(f^{\prime}(x)+\frac{t^{\alpha} f_{1}^{\prime}(x)}{\alpha}+\frac{t^{2 \alpha} f_{2}^{\prime}(x)}{2 \alpha^{2}}\right)-3\left(f(x)+\frac{t^{\alpha} f_{1}(x)}{\alpha}+\frac{t^{2 \alpha} f_{2}(x)}{2 \alpha^{2}}\right)^{2} \\
& -3\left(g(x)+\frac{t^{\alpha} g_{1}(x)}{\alpha}+\frac{t^{2 \alpha} g_{2}(x)}{2 \alpha^{2}}\right)^{2} \times\left(g^{\prime}(x)+\frac{t^{\alpha} g_{1}^{\prime}(x)}{\alpha}+\frac{t^{2 \alpha} g_{2}^{\prime}(x)}{2 \alpha^{2}}\right) \\
& +3\left(f^{\prime}(x)+\frac{t^{\alpha} f_{1}^{\prime}(x)}{\alpha}+\frac{t^{2 \alpha} f_{2}^{\prime}(x)}{2 \alpha^{2}}\right)\left(g^{\prime}(x)+\frac{t^{\alpha} g_{1}^{\prime}(x)}{\alpha}+\frac{t^{2 \alpha} g_{2}^{\prime}(x)}{2 \alpha^{2}}\right) \\
& +\left(g(x)+\frac{t^{\alpha} g_{1}(x)}{\alpha}+\frac{t^{2 \alpha} g_{2}(x)}{2 \alpha^{2}}\right)\left(f^{\prime \prime}(x)+\frac{t^{\alpha} f_{1}^{\prime \prime}(x)}{\alpha}+\frac{t^{\alpha} f_{2}^{\prime \prime}(x)}{2 \alpha^{2}}\right) \\
& +g^{(3)}(x)+\frac{t^{\alpha} g_{1}^{(3)}(x)}{\alpha}+\frac{t^{2 \alpha} g_{2}^{(3)}(x)}{2 \alpha^{2}} .
\end{aligned}
$$

Now, applying $T_{\alpha}$ on both sides of $\operatorname{Resu}_{2}(x, t)$ and $\operatorname{Resv}_{2}(x, t)$ and equating to 0 for $t=0$ gives: 


$$
\begin{gathered}
f_{2}(x)=-\frac{\alpha^{5}}{2 \alpha^{5}-9 t^{5 \alpha} g_{2} g_{2}^{\prime}}\left(12 f f_{1} f^{\prime}-12 g g_{1} f^{\prime}-12 g f_{1} g^{\prime}-12 f g_{1} g^{\prime}+6 f^{2} f_{1}^{\prime}-6 g^{2} f_{1}^{\prime}-12 f g g_{1}^{\prime}-12 g^{\prime} g_{1}^{\prime}\right) \\
-\frac{\alpha^{5}}{2 \alpha^{5}-9 t^{5 \alpha} g_{2} g_{2}^{\prime}}\left(-6 g_{1} g^{\prime \prime}-6 g g_{1}^{\prime \prime}-f_{1}^{(3)}-12 f g g_{1}^{\prime}-12 g^{\prime} g_{1}^{\prime}-6 g_{1} g^{\prime \prime}-6 g g_{1}^{\prime \prime}-f_{1}^{(3)}\right), \\
g_{2}(x)=6 g f_{1} f^{\prime}+6 f g_{1} f^{\prime}+6 f f_{1} g^{\prime}-6 g g_{1} g^{\prime}+6 f g f_{1}^{\prime}-3 g^{\prime} f_{1}^{\prime} \\
+3 f^{2} g_{1}^{\prime}-3 g^{2} g_{1}^{\prime}-3 f^{\prime} g_{1}^{\prime}-3 g_{1} f^{\prime \prime}-3 g f_{1}^{\prime \prime}-g_{1}^{(3)} .
\end{gathered}
$$

Therefore the $2 n d$ RPS approximate solutions of time-fractional new coupled $\mathrm{mKdV}$ equation is obtained as:

$$
\begin{gathered}
u_{2}(x, t)=f+\frac{t^{\alpha} f_{1}}{\alpha}-\frac{t^{2 \alpha} \alpha^{3}}{2\left(2 \alpha^{5}-9 t^{5 \alpha} g_{2} g_{2}^{\prime}\right)}\left(12 f f_{1} f^{\prime}-12 g g_{1} f^{\prime}-12 g f_{1} g^{\prime}-12 f g_{1} g^{\prime}+6 f^{2} f_{1}^{\prime}\right) \\
-\frac{t^{2 \alpha} \alpha^{3}}{2\left(2 \alpha^{5}-9 t^{5 \alpha} g_{2} g_{2}^{\prime}\right)}\left(-6 g^{2} f_{1}^{\prime}-12 f g g_{1}^{\prime}-12 g^{\prime} g_{1}^{\prime}-6 g_{1} g^{\prime \prime}-6 g g_{1}^{\prime \prime}-f_{1}^{(3)}\right), \\
v_{2}(x, t)=g(x)+\frac{t^{\alpha} g_{1}(x)}{\alpha}+\frac{t^{2 \alpha}}{2 \alpha^{2}}\left(6 g f_{1} f^{\prime}+6 f g_{1} f^{\prime}+6 f f_{1} g^{\prime}-6 g g_{1} g^{\prime}+6 f g f_{1}^{\prime}\right) \\
+\frac{t^{2 \alpha}}{2 \alpha^{2}}\left(-3 g^{\prime} f_{1}^{\prime}+3 f^{2} g_{1}^{\prime}-3 g^{2} g_{1}^{\prime}-3 f^{\prime} g_{1}^{\prime}-3 g_{1} f^{\prime \prime}-3 g f_{1}^{\prime \prime}-g_{1}^{(3)}\right) .
\end{gathered}
$$

In the same manner, we apply the same procedure for $n=3$ to obtain the following results.

$$
\begin{aligned}
f_{3}(x)= & -6 f_{1}^{2} f^{\prime}-6 f f_{2} f^{\prime}+6 g_{1}^{2} f^{\prime}+6 g g_{2} f^{\prime}+6 g f_{2} g^{\prime}+12 f_{1} g_{1} g^{\prime}+6 f g_{2} g^{\prime} \\
& -12 f f_{1} f_{1}^{\prime}+12 g g_{1} f_{1}^{\prime}-3 f^{2} f_{2}^{\prime}+3 g^{2} f_{2}^{\prime}+12 g f_{1} g_{1}^{\prime}+12 f g_{1} g_{1}^{\prime} \\
& +6 g_{1}^{\prime 2}+6 f g g_{2}^{\prime}+6 g^{\prime} g_{2}^{\prime}+3 g_{2} g^{\prime \prime}+6 g_{1} g_{1}^{\prime \prime}+3 g g_{2}^{\prime \prime}+\frac{1}{2} f_{2}^{(3)},
\end{aligned}
$$$$
g_{3}(x)=6 g f_{2} f^{\prime}+12 f_{1} g_{1} f^{\prime}+6 f g_{2} f^{\prime}+6 f_{1}^{2} g^{\prime}+6 f f_{2} g^{\prime}-6 g_{1}^{2} g^{\prime}-6 g g_{2} g^{\prime}
$$$$
+12 g f_{1} f_{1}^{\prime}+12 f g_{1} f_{1}^{\prime}+6 f g f_{2}^{\prime}-3 g^{\prime} f_{2}^{\prime}+12 f f_{1} g_{1}^{\prime}-12 g g_{1} g_{1}^{\prime}-6 f_{1}^{\prime} g_{1}^{\prime}
$$$$
+3 f^{2} g_{2}^{\prime}-3 g^{2} g_{2}^{\prime}-3 f^{\prime} g_{2}^{\prime}-3 g_{2} f^{\prime \prime}-6 g_{1} f_{1}^{\prime \prime}-3 g f_{2}^{\prime \prime}-g_{2}^{(3)},
$$

$$
\begin{aligned}
u_{3}(x, t)= & f(x)+\frac{t^{\alpha} f_{1}}{\alpha}+\frac{t^{2 \alpha} f_{2}}{2 \alpha^{2}}+\frac{t^{3 \alpha}}{6 \alpha^{3}}\left(-6 f_{1}^{2} f^{\prime}-6 f f_{2} f^{\prime}+6 g_{1}^{2} f^{\prime}+6 g g_{2} f^{\prime}+6 g f_{2} g^{\prime}+12 f_{1} g_{1} g^{\prime}\right) \\
& +\frac{t^{3 \alpha}}{6 \alpha^{3}}\left(+6 f g_{2} g^{\prime}-12 f f_{1} f_{1}^{\prime}+12 g g_{1} f_{1}^{\prime}-3 f^{2} f_{2}^{\prime}+3 g^{2} f_{2}^{\prime}+12 g f_{1} g_{1}^{\prime}+12 f g_{1} g_{1}^{\prime}\right) \\
& +\frac{t^{3 \alpha}}{6 \alpha^{3}}\left(+6 g_{1}^{\prime 2}+6 f g g_{2}^{\prime}+6 g^{\prime} g_{2}^{\prime}+3 g_{2} g^{\prime \prime}+6 g_{1} g_{1}^{\prime \prime}+3 g g_{2}^{\prime \prime}+\frac{1}{2} f_{2}^{(3)}\right),
\end{aligned}
$$

$$
\begin{aligned}
v_{3}(x, t)= & g+\frac{t^{\alpha} g_{1}}{\alpha}+\frac{t^{2 \alpha} g_{2}}{2 \alpha^{2}}+\frac{t^{3 \alpha}}{6 \alpha^{3}}\left(6 g f_{2} f^{\prime}+12 f_{1} g_{1} f^{\prime}+6 f g_{2} f^{\prime}+6 f_{1}^{2} g^{\prime}+6 f f_{2} g^{\prime}-6 g_{1}^{2} g^{\prime}-6 g g_{2} g^{\prime}\right) \\
& +\frac{t^{3 \alpha}}{6 \alpha^{3}}\left(12 g f_{1} f_{1}^{\prime}+12 f g_{1} f_{1}^{\prime}+6 f g f_{2}^{\prime}-3 g^{\prime} f_{2}^{\prime}+12 f f_{1} g_{1}^{\prime}-12 g g_{1} g_{1}^{\prime}-6 f_{1}^{\prime} g_{1}^{\prime}\right) \\
& +\frac{t^{3 \alpha}}{6 \alpha^{3}}\left(3 f^{2} g_{2}^{\prime}-3 g^{2} g_{2}^{\prime}-3 f^{\prime} g_{2}^{\prime}-3 g_{2} f^{\prime \prime}-6 g_{1} f_{1}^{\prime \prime}-3 g f_{2}^{\prime \prime}-g_{2}^{(3)}\right) .
\end{aligned}
$$


In Tables 1-2, the fourth-order approximate RPSM solutions of time-fractional new coupled mKdV equation are compared numerically with the exact solutions

$$
\begin{gathered}
u(x, t)=\frac{1}{2} \sqrt{-\sigma} \tanh \left(\sqrt{-\sigma}\left(x-\frac{\sigma t^{\alpha}}{2 \alpha}\right)\right), \\
v(x, t)=-\frac{1}{2} \sqrt{-\sigma} \tanh \left(\sqrt{-\sigma}\left(x-\frac{\sigma t^{\alpha}}{2 \alpha}\right)\right) .
\end{gathered}
$$

TABLE 1. Numerical results of third order RPSM $\left(u_{3}(x, t)\right)$ solutions with absolute errors for $\sigma=-1 / 2$ and $t=0.1$.

\begin{tabular}{|c|ccc|ccc|ccc|}
\hline & \multicolumn{3}{|c|}{$\alpha=0.25$} & \multicolumn{3}{c|}{$\alpha=0.50$} & \multicolumn{3}{c|}{$\alpha=0.75$} \\
\hline$x$ & RPSM & Exact & Abs. Error & RPSM & Exact & Abs. Error & $R P S M$ & Exact & Abs. Error \\
\hline 0.0 & 0.132820 & 0.133616 & $7.96289 \mathrm{E}-4$ & 0.039363 & 0.039364 & $8.19389 \mathrm{E}-7$ & 0.014810 & 0.014810 & $6.09402 \mathrm{E}-9$ \\
0.1 & 0.160405 & 0.154454 & $5.95048 \mathrm{E}-3$ & 0.063818 & 0.063821 & $3.06690 \mathrm{E}-6$ & 0.039651 & 0.039651 & $5.64029 \mathrm{E}-8$ \\
0.2 & 0.183978 & 0.174045 & $9.93250 \mathrm{E}-3$ & 0.087657 & 0.087662 & $5.16170 \mathrm{E}-6$ & 0.064102 & 0.064102 & $1.02078 \mathrm{E}-7$ \\
0.3 & 0.201688 & 0.192320 & $9.36789 \mathrm{E}-3$ & 0.110677 & 0.110684 & $6.98854 \mathrm{E}-6$ & 0.087935 & 0.087935 & $1.39622 \mathrm{E}-7$ \\
0.4 & 0.214072 & 0.209244 & $4.82823 \mathrm{E}-3$ & 0.132701 & 0.132709 & $8.40691 \mathrm{E}-6$ & 0.110946 & 0.110946 & $1.66603 \mathrm{E}-7$ \\
0.5 & 0.223533 & 0.224810 & $1.27678 \mathrm{E}-3$ & 0.153588 & 0.153598 & $9.28657 \mathrm{E}-6$ & 0.132959 & 0.132959 & $1.81898 \mathrm{E}-7$ \\
0.6 & 0.232774 & 0.239038 & $6.26428 \mathrm{E}-3$ & 0.173234 & 0.173243 & $9.55621 \mathrm{E}-6$ & 0.153833 & 0.153833 & $1.85693 \mathrm{E}-7$ \\
0.7 & 0.243403 & 0.251971 & $8.56755 \mathrm{E}-3$ & 0.191566 & 0.191575 & $9.23384 \mathrm{E}-6$ & 0.173464 & 0.173464 & $1.79282 \mathrm{E}-7$ \\
0.8 & 0.255536 & 0.263664 & $8.12801 \mathrm{E}-3$ & 0.208547 & 0.208556 & $8.42239 \mathrm{E}-6$ & 0.191780 & 0.191780 & $1.64722 \mathrm{E}-7$ \\
0.9 & 0.268286 & 0.274188 & $5.90160 \mathrm{E}-3$ & 0.224172 & 0.224179 & $7.27695 \mathrm{E}-6$ & 0.208745 & 0.208745 & $1.44442 \mathrm{E}-7$ \\
1.0 & 0.280523 & 0.283619 & $3.09595 \mathrm{E}-3$ & 0.238457 & 0.238463 & $5.96287 \mathrm{E}-6$ & 0.224352 & 0.224352 & $1.20880 \mathrm{E}-7$ \\
\hline
\end{tabular}

Absolute errors are presented for $\alpha=0.25, \alpha=0.50$ and $\alpha=0.75$. The results indicate that as the $x$ values increase the absolute errors also increase. Besides, as the $\alpha$ values increase, the absolute errors decrease. Also the Tables 1 and 2 show competitive solutions of the RPSM with highly approximate results. Moreover, in figures, the surface plots of the approximate solutions are illustrated for $\alpha=0.25, \alpha=0.50$ and $\alpha=0.75$.

TABLE 2. Numerical results of third order RPSM $\left(v_{3}(x, t)\right)$ solutions with absolute errors for $\sigma=-1 / 2$ and $t=0.1$.

\begin{tabular}{|c|ccc|ccc|ccc|}
\hline & \multicolumn{3}{|c|}{$\alpha=0.25$} & \multicolumn{3}{c|}{$\alpha=0.50$} & \multicolumn{3}{c|}{$\alpha=0.75$} \\
\hline$x$ & RPSM & Exact & Abs. Error & RPSM & Exact & Abs. Error & RPSM & Exact & Abs. Error \\
\hline 0.0 & -0.133176 & -0.133616 & $4.40433 \mathrm{E}-4$ & -0.039363 & -0.039364 & $8.19364 \mathrm{E}-7$ & -0.014810 & -0.014810 & $6.09402 \mathrm{E}-9$ \\
0.1 & -0.154147 & -0.154454 & $3.07373 \mathrm{E}-4$ & -0.063818 & -0.063821 & $3.31577 \mathrm{E}-6$ & -0.039651 & -0.039651 & $5.64978 \mathrm{E}-8$ \\
0.2 & -0.173537 & -0.174045 & $5.07560 \mathrm{E}-4$ & -0.087657 & -0.087662 & $5.55303 \mathrm{E}-6$ & -0.064102 & -0.064102 & $1.02223 \mathrm{E}-7$ \\
0.3 & -0.191150 & -0.192320 & $1.16995 \mathrm{E}-3$ & -0.110676 & -0.110684 & $7.36827 \mathrm{E}-6$ & -0.087935 & -0.087935 & $1.39755 \mathrm{E}-7$ \\
0.4 & -0.207176 & -0.209244 & $2.06771 \mathrm{E}-3$ & -0.132701 & -0.132709 & $8.64380 \mathrm{E}-6$ & -0.110946 & -0.110946 & $1.66675 \mathrm{E}-7$ \\
0.5 & -0.222035 & -0.224810 & $2.77502 \mathrm{E}-3$ & -0.153588 & -0.153598 & $9.32228 \mathrm{E}-6$ & -0.132959 & -0.132959 & $1.81889 \mathrm{E}-7$ \\
0.6 & -0.23609 & -0.239038 & $2.94836 \mathrm{E}-3$ & -0.173234 & -0.173243 & $9.41358 \mathrm{E}-6$ & -0.153833 & -0.153833 & $1.85615 \mathrm{E}-7$ \\
0.7 & -0.249455 & -0.251971 & $2.51534 \mathrm{E}-3$ & -0.191566 & -0.191575 & $8.98895 \mathrm{E}-6$ & -0.173464 & -0.173464 & $1.79169 \mathrm{E}-7$ \\
0.8 & -0.261997 & -0.263664 & $1.66669 \mathrm{E}-3$ & -0.208547 & -0.208556 & $8.16298 \mathrm{E}-6$ & -0.191780 & -0.191780 & $1.64611 \mathrm{E}-7$ \\
0.9 & -0.273478 & -0.274188 & $7.09964 \mathrm{E}-4$ & -0.224172 & -0.224179 & $7.06952 \mathrm{E}-6$ & -0.208745 & -0.208745 & $1.44359 \mathrm{E}-7$ \\
1.0 & -0.283708 & -0.283619 & $8.86102 \mathrm{E}-5$ & -0.238458 & -0.238463 & $5.83883 \mathrm{E}-6$ & -0.224352 & -0.224352 & $1.20836 \mathrm{E}-7$ \\
\hline
\end{tabular}


(a)

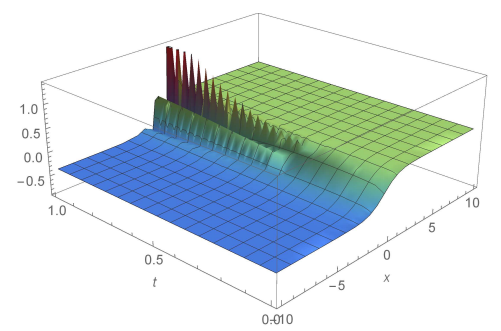

(b)

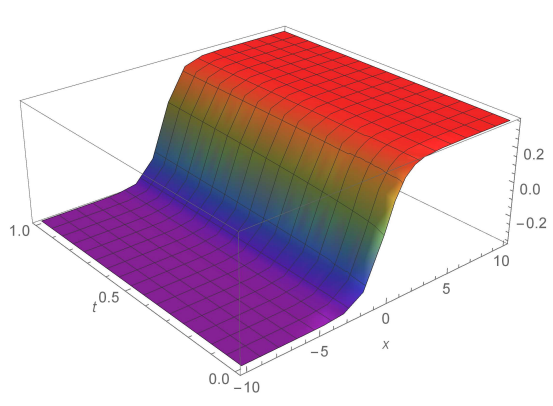

(c)

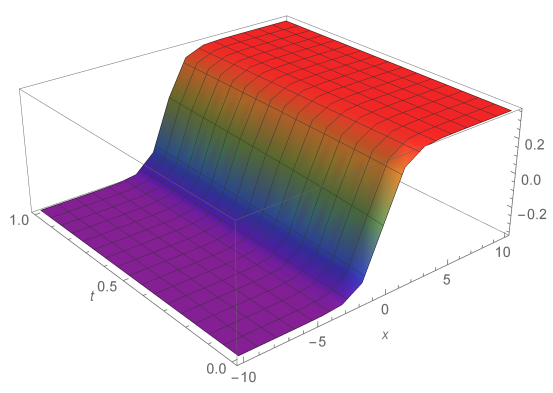

Figure 1. The surface plots of $u_{3}(x, t)$ for $\sigma=-1 / 2$ and $t=0.1$ and for a.) $\alpha=0.25, \mathrm{~b}$.) $\alpha=0.50$, c.) $\alpha=0.75$. 
(a)

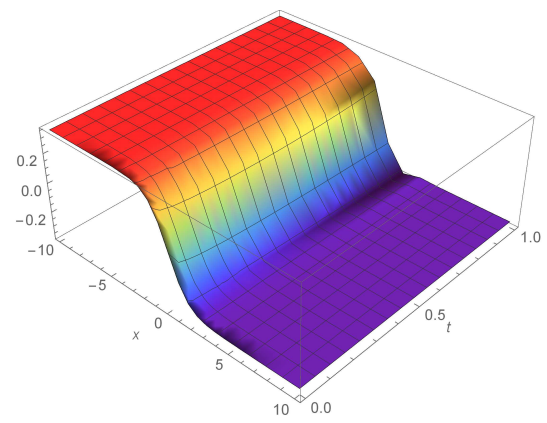

(b)

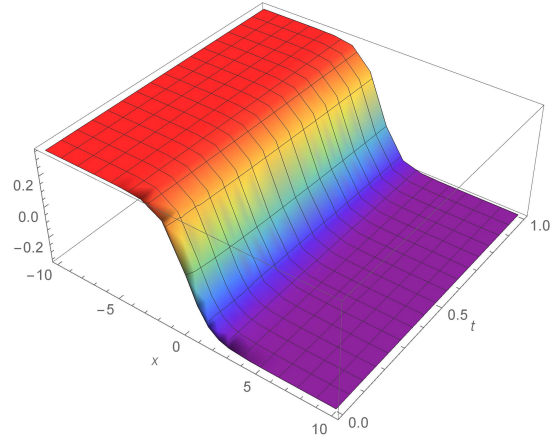

(c)

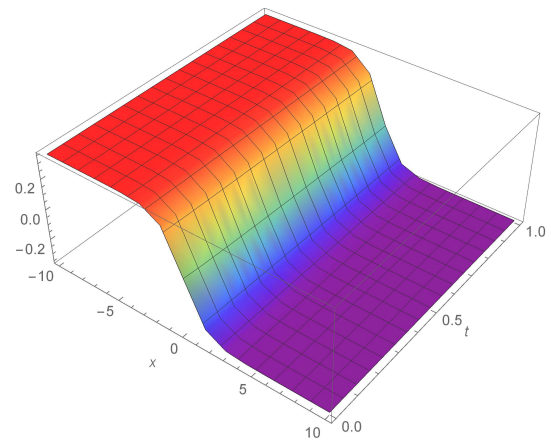

Figure 2. The surface plots of $v_{3}(x, t)$ for $\sigma=-1 / 2$ and $t=0.1$ and for a.) $\alpha=0.25, \mathrm{~b}$.) $\alpha=0.50$, c.) $\alpha=0.75$. 


\section{Conclusion}

In this paper, firstly the exact solutions of the nonlinear time-fractional new coupled $\mathrm{mKdV}$ equations are obtained by the sub-equation method. Then the approximate solution of the model is demonstrated by the residual power series method. The fractional derivatives in the solution procedure is taken in the conformable sense. By the proposed methods and conformable fractional derivative definition, it is shown dependable ways of obtaining exact and approximate solutions for nonlinear fractional partial differential equations. Approximate solutions are compared with the exact solutions to show the reliability of the methods. Absolute errors are given with approximate and exact solutions with the help of figures and tables. Therefore, we can conclude that the methods are very effective tools for FDEs arising in different branches of applied sciences.

\section{REFERENCES}

[1] T. Abdeljawad, On conformable fractional calculus, J. Comput. Appl. Math. 279 (2015), 57-66.

[2] R. S.Ahmad, An analytical solution of the fractional Navier-Stokes equation by residual power series method, Ph.D Thesis, Zarqa University, 2015.

[3] T.Y. Alabsi, Numerical study of laser beam, M.S. thesis, Zarqa University, 2017.

[4] M. Alquran, Marwan, Analytical solutions of fractional foam drainage equation by residual power series method, Math. Sci. 8 (2014), 153-160.

[5] M. Alquran, Analytical solution of time-fractional two-component evolutionary system of order 2 by residual power series method, J. Appl. Anal. Comput. 5 (2015), 589-599.

[6] O. A. Arqub, Series solution of fuzzy differential equations under strongly generalized differentiability, J. Adv. Res. Appl. Math. 5 (2013), 31-52.

[7] O.A. Arqub, A. El-Ajou, A. Bataineh, I. Hashim, A representation of the exact solution of generalized Lane-Emden equations using a new analytical method, Abstr. Appl. Anal. 2013 (2013), Article ID 378593, 10 pages.

[8] A. Atangana, D. Baleanu, A. Alsaedi, New properties of conformable derivative, Open Math. 13 (2015), 889-898.

[9] R. L. Bagley, P. J. Torvik, A theoretical basis for the application of fractional calculus to viscoelasticity, J. Rheology 27 (1983), $201-210$.

[10] K. Balachandran, J. Y Park, J.J. Trujillo, Controllability of nonlinear fractional dynamical systems, Nonlinear Anal., Theory Methods Appl. 75 (2012), 1919-1926.

[11] A. Bekir, c E. Aksoy, A. C. Cevikel, Exact solutions of nonlinear time fractional partial differential equations by sub-equation method, Math. Methods Appl. Sci. 38 (2015), 2779-2784.

[12] D. B. Cao, J. R. Yan, Y. Zhang, Exact solutions for a new coupled MKdV equations and a coupled KdV equations, Phys. Lett. A, 297 (2002), 68-74.

[13] M. Caputo, Linear models of dissipation whose Q is almost frequency independent-II, Geophys. J. Int. 13 (1967), $529-539$.

[14] Y. Cenesiz, A. Kurt, New fractional complex transform for conformable fractional partial differential equations, J. Appl. Math. Stat. Inf. $12(2016), 41-47$.

[15] S. Das, Functional fractional calculus, Springer, 2011.

[16] K. Diethelm, The analysis of fractional differential equations, Springer, 2010.

[17] J. S. Duan, R. Rach, D., Baleanu, A.M. Wazwaz, A review of the Adomian decomposition method and its applications to fractional differential equations, Commun. Fract. Calc. 3 (2012), 73-99.

[18] A. El-Ajou, O. A. Arqub, Z. A. Zhour, S. Momani, New results on fractional power series: theories and applications, Entropy, 15 (2013), 5305-5323.

[19] T.J. Freeborn, A survey of fractional-order circuit models for biology and biomedicine, IEEE Journal on Emerging and Selected Topics in Circuits and Systems 3 (2013), 416-424. 
[20] H. Jafari, V. Daftardar-Gejji, Solving linear and nonlinear fractional diffusion and wave equations by Adomian decomposition, Appl. Math. Comput. 180 (2006), 488-497.

[21] H.M. Jaradat, S. Al-Shara, Q.J. Khan, M. Alquran, Al-Khaled, Analytical solution of time-fractional Drinfeld-Sokolov-Wilson system using residual power series method, IAENG Int. J. Appl. Math, 46 (2016), 64-70.

[22] R. Khalil, M. Al Horani, A. Yousef, M. Sababheh, A new definition of fractional derivative, J. Comput. Appl. Math. 264 (2014), 65-70.

[23] A. Kumar, S. Kumar, M. Singh, Residual power series method for fractional Sharma-Tasso-Olever equation, Commun. Numer. Anal, 10 (2016).

[24] A. Kurt, O. Tasbozan, D. Baleanu, New solutions for conformable fractional Nizhnik-Novikov-Veselov system via $G^{\prime} / G$ expansion method and homotopy analysis methods, Opt. Quant. Electr. 49 (2017), 333.

[25] A. Kurt, O. Tasbozan, Y. Cenesiz, Homotopy analysis method for conformable Burgers-Korteweg-de Vries equation, Bull. Math. Sci. Appl. 17 (2016), 17-23.

[26] W. Malfliet, Solitary wave solutions of nonlinear wave equations, Amer. J. Phys. 60 (1992), 650-654.

[27] M. M. Meerschaert, C. Tadjeran, Finite difference approximations for two-sided space-fractional partial differential equations, Appl. Numer. Math. 56 (2006), 80-90.

[28] S. Momani, Z. Odibat, Analytical approach to linear fractional partial differential equations arising in fluid mechanics, Phys. Lett. A, 355 (2006), 271-279.

[29] Z.M. Odibat, S. Momani, Application of variational iteration method to nonlinear differential equations of fractional order, Int. J. Nonlinear Sci. Numer. Simul. 7 (2006), 27-34.

[30] M. Şenol, İ.T. Dolapci, On the Perturbation-Iteration Algorithm for fractional differential equations, J. King Saud Univ., Sci. s28 (2016), 69-74.

[31] M. Şenol, M. Alquran, H.D. Kasmaei, On the comparison of perturbation-iteration algorithm and residual power series method to solve fractional Zakharov-Kuznetsov equation, Results Phys. 9 (2018), 321-327.

[32] Y. Takeuchi, Y. Yoshimoto, R. Suda, Second order accuracy finite difference methods for space-fractional partial differential equations, J. Comput. Appl. Math. 320 (2017), 101-119.

[33] O. Tasbozan, Y. Çenesiz, A. Kurt, New solutions for conformable fractional Boussinesq and combined KdV-mKdV equations using Jacobi elliptic function expansion method, Eur. Phys. J. Plus, 131 (2016), 244.

[34] X.J. Yang, H. M. Srivastava, C. Cattani, Local fractional homotopy perturbation method for solving fractal partial differential equations arising in mathematical physics, Romanian Reports in Physics, 67 (3) (2015), 752-761.

[35] C. Yeroglu, C., B. Senol, Investigation of robust stability of fractional order multilinear affine systems: 2q-convex parpolygon approach, Syst. Control Lett. 62 (2013), 845-855.

[36] S. Zhang, H. Q. Zhang, Fractional sub-equation method and its applications to nonlinear fractional PDEs, Phys. Lett. A, 375 (2011), 1069-1073. 